\section{Congenital Dislocation of the Hip}

SIR,-Mr. A. W. Fowler (9 December, p. 618) is inaccurate in his description of Ortolani's test, which he describes as " an attempt to assess the hip by manipulating the shaft of the femur," rather than by direct digital palpation of the hip as described by Barlow. ${ }^{1}$ In his monograph La Lussazione Congenita dell'Anca published in 1948 Ortolani ${ }^{2}$ clearly describes the placing of the thumb of the examining hand over the medial aspect of the hip, with the index finger on the greater trochanter pressing in a latero-medial direction, while the palm keeps the knee flexed. There are accompanying photographs which emphasize this practical point unmistakably. Furthermore, in his film "Diagnosis, Prophylaxis, and Treatment of Congenital Hip Dislocation at Birth" Professor Ortolani again clearly describes and demonstrates this technique, and this was how he demonstrated it to me when I visited him in Ferrara in May 1960. He most definitely does recommend the use of his test on the newborn child.

Mr. T. G. Barlow is thus correct in describing his test as a modification of Ortolani's test, and the writer of your leading article (18 November, p. 371) does valuable service in making such a clear and simple interpretation of it. This is a test we want the general practitioner and the midwife to perform with confidence on the newborn child. In my experience many of them are confused by the varied descriptions of what they should do, and by the various interpretations of the "clicks" and "clonks" which they may find, with the result that they lack confidence in the test. They should read, and reread, the relevant paragraph in that leading article.-I am, etc.,

Robert Jones and Agnes Hunt

$$
\text { G. C. Slee. }
$$

Orthopaedic Hospital,

\section{REFERENCES}

I Barlow, T. G., Y. Bone It Surg., 1962, 44B, 292. 1948.' Cappelli, Bologna.

\section{Hip Arthroplasty and Haemophilia}

SIR,-The article on hip arthroplasty in a haemophiliac (2 December, p. 531) calls for a number of comments regarding both the haematological and orthopaedic management of the case.

I work at Lewisham Hospital, where our haematology department has a sizable clinic for haemophiliacs with about 100 regular attenders. With the aid of my haematological colleagues I have performed a number of minor and major orthopaedic procedures-for example, a below-knee amputation and arthrodesis of an ankle joint. However, they insist that operations on haemophiliacs should be avoided if circulating anticoagulants are present, a point which should have been stressed in the article. About two or three years ago a patient was sent to me from Liverpool for a meniscectomy. We did not carry this out, because circulating antibodies were demonstrated.

I feel most unhappy about the treatment of what is described as a transverse fracture of the femoral neck. Unless I misunderstand the article, the patient was subjected to supervised inactivity for 16 weeks, after which a Thompson prosthesis was cemented in under cover of antihaemophilic globulin concentrate. In my opinion the simplest line of approach would have been immediate mobilization with the aid of a calliper. In many instances the femoral neck becomes rubbed away completely, and the end result is a Girdlestone operation, but without surgery. However, if the fracture was such that this simple treatment would not have worked, internal fixation should have been performed, using either a cannulated nail or preferably a screw. It is possible to do this through a $1-1 \frac{1}{2}$-in. $(2.5-3.5-\mathrm{cm}$.) incision by percutaneous insertion of the guide-wires. The position of the screw should be near vertical It should pass through the centre of the femoral head and rest on the inferior cortex of the distal fragment. The patient is only 21 years old and this treatment might well have been successful. In case of failure a Girdlestone operation can always be performed as a salvage procedure. The treatment actually adopted carries two risks.

The first risk is that of late sepsis. Having seen a sinus develop in a patient 18 months after cementing in an Austin Moore prosthesis, I should certainly not have used surgical Simplex P. It would have been better to use an Austin Moore implant and rely for fixation on bone growing across the two fenestrations. The development of a sinus would pose an almost insoluble problem in this particular patient.

The second risk is mechanical failure of the acetabulum. One of the methods of hardness testing employed by engineers consists in squeezing a hardened steel ball into the material to be tested under a fixed load. This is the basis of the Brinell test, which this patient now carries out on his pelvis with every step he takes. Eventually, the articular cartilage of the acetabulum will yield and his hip will become painful. It is anticipated that in a few years' time either acetabular osteophytes will be present or the prosthesis will have sunk deeply into the pelvis.

I hope the authors will publish a follow-up report in five years' time.-I am, etc.

Lewisham Hospital,
London S.E.13.

A. C. Bingold.

$$
\text { A. Bingord. }
$$

\section{Mushroom-worker's Lung}

SIR,-I have noted Dr. A. Sakula's comments (9 December, p. 619) regarding the case described in my letter (18 November, p. 421). My patient did resume work at the mushroom farm in August 1965, and he is still employed there. He has had no recurrence of the respiratory symptoms. Corticosteroid treatment ceased in November 1965.

Originally I would like to have diagnosed him as a case of mushroom-worker's lung, and after Dr. Sakula's paper was published (16 September, p. 708) I reappraised his case. However, the weight of all the evidence, including that provided by the excised scalene node, appeared to be strongly in favour of Boeck's sarcoidosis. While accepting that lesions indistinguishable from Boeck's sarcoid may occasionally be observed in lymph nodes draining a malignant neoplasm, no suggestion has been found that allergic conditions in the lung produce similar changes in the drainage glands. It is tempting to think that I may have been dealing with the first case of mushroom-worker's lung in which such an extensiou was actually noted, but it seems unjustifiable to claim this on the basis of the facts available. Also, it is probably relevant that my patient did have ocular symptoms, diagnosed as being due to episcleritis, early in his illness. This feature seemed more in accordance with a sarcoid pattern than one of allergic alveolitis.

The fact that neither of Dr. Sakula's cases (1) and (2) had any other farming experience certainly strengthens the likelihood of an association between their respiratory symptoms and their work with mushrooms, and it seems almost certain that there was a connexion. Nevertheless, I would like to stress that it is possible to be misled when attempting to establish a clear-cut relationship between cause and effect in illnesses of this type and to quote one illustrative example. The patient, a woman, was referred because of progressive dyspnoea. Her chest radiograph showed evidence of bilateral pulmonary fibrosis. As she had worked with poultry in deep-litter over a period of years and her serum gave reactions compatible with farmer's lung, it was accepted that this was the cause of her lung damage. At a much later date, however, after reports appeared about bird-fancier's lung, it was ascertained that she had also been in contact with pigeons and a budgerigar belonging to her young son, and strong avian precipitins were found in her blood. The exact aetiological role of each of the different types of allergens in her illness can therefore be only a matter of conjecture. Now I take the view that the avian ones were probably the major factor and that her pulmonary disease is in fact really an example of bird-fancier's lung and not farmer's lung, as was first thought. -I am, etc.,

$$
\begin{aligned}
& \text { Coleraine, } \\
& \text { N. Ireland. } \\
& \text { C. T. B. Adams. }
\end{aligned}
$$

(n)

\section{Prevention of Rh-haemolytic Disease}

SIR,-Since my lecture (7 October, p. 7) the numbers which I reported in the various trials have increased and the totals at six months after delivery are now, so far as I know: 80 immunized out of 860 controls and 2 immunized out of 1,113 treated. The discrepancy in the American figures that I sent to Dr. Sheilagh Murray (16 December, p. 682) was due to the fact that they were obtained from two different sources (a difficulty which Dr. J. G. Robertson has now kindly clarified), and that in the German ones to a misunderstanding due to language. The latest figures for subsequent $\mathrm{Rh}$-positive pregnancies are (again so far as I know) 19 immunized out of 79 controls (who were not immunized at six months) and two immunized out of 87 treated. There have therefore been four failures out of 1,113 treated, two developing antibodies six months after delivery and two during the subsequent pregnancy. The figure to compare with this is 99 antibodies out of 860 controls.

There is also new information about two additional large-scale trials. The first, from Winnipeg, ${ }^{2}$ although differing in some respects from our own, gives much the same information-that is, the giving of antiD gammaglobulin appears to be successful in preventing immunization. The second is from St. Louis. Hamilton (personal com- 
munication and footnote ${ }^{2}$ ) states that he has successfully treated in the past six years, using $10 \mathrm{ml}$. of blood plasma obtained from sensitized $\mathrm{Rh}$-negative women (with particular precautions to avoid jaundice), over 500 women and no case of $\mathrm{Rh}$-immunization has occurred. Thirty-eight of his patients have received a second dose of plasma after a subsequent pregnancy and four a third one, and no unfavourable reactions to the treatment have been observed. On the other hand, of the 88 controls 16 showed evidence of immunization in the next observed pregnancy.

The experience of giving either plasma or gammaglobulin to volunteers or parturient women now goes back more than seven years, and in none of the trials has rheumatoid arthritis been reported, though it has been looked for, particularly by Hamilton. Any fear of this, therefore, seems likely to be a theoretical rather than an actual problem. In fact there seems no escaping the conclusion that the data at the present time suggest that a considerable number of subjects have been prevented from becoming immunized and are none the worse for the treatment. This does not mean that there are no problems, and much research is going on By far the most important practical point concerns dosage, and there is evidence that in some cases not enough gammaglobulin is being given to women who have had a very large (that is, of the order of $50 \mathrm{ml}$. or more) foeto-maternal haemorrhage. ${ }^{3}$ - $-\mathrm{I}$ am. etc.,

Department of Medicine, C. A. Clarke. The University,
Liverpool.

ReFERENCES

' Zipursky, A., and Israels, L. G., Canad. med. Ass. Y., 1967, 97, 1245

International Medical Tribune of Great Britain. 9 November 1967 , p. 8

Woodrow, J. C., personal communication.

S. Bowley, Brit. med. C. .'. (in press).

\section{Incidence of Diverticulosis}

SIR,-The article by Dr. O. N. Manousos and others (23 September, p. 762) on the prevalence of colonic diverticulosis is welcome because it emphasizes the high incidence of this condition in the general (Western) population. That such an emphasis is necessary is highlighted by the fact that the last two leading articles in the B.M.F. on this subject (23 September, 1967, p. 751, and 14 May, 1966, p. 1186) quote the figures of Dearlove's post-mortem series, ${ }^{1}$ where an incidence of diverticulosis of nil at age 40 to $7 \%$ at age 70 was found. Knowledge of the true incidence is essential because of the current widespread attribution of many symptoms-abdominal pain, diarrhoea, constipation, and bleeding to diverticulosis or its precursor state. Although such an association is undoubtedly warranted in many cases on present evidence, if the true incidence of diverticulosis is, for instance, $50 \%$ of the population at risk, the possibility of chance association must be given due consideration.

Dr. Manousos and colleagues' study is particularly important in that an asymptomatic group were studied. However, it is likely that barium studies will considerably underestimate the incidence of the disease, as it is well known that muscular activity will frequently prevent filling of diverticula during qarium enema and that follow-through studies will miss many early cases, as diverticula less than $5 \mathrm{~mm}$. do not retain their contents for long periods.

As diverticulosis is a relatively rare cause of death (500 cases per year in Great Britain) and diverticular disease has not been shown to be associated with any common cause of hospitalization or death, careful studies on consecutive necropsies should give the most reliable results. We have carried out such a study on 200 consecutive unselected necropsy colons. ${ }^{2}$ The results are being presented more fully elsewhere, but, briefly, it revealed an incidence of diverticulosis of $47.5 \%$ of patients over the age of 50 . This figure was made up of $36 \%$ in the sixth and seventh decades and $56 \%$ over the age of 70 , while there was an incidence of $9 \%$ under the age of $50.14 \%$ of patients with diverticulosis had less than five (usually quite small) diverticula-a group likely to be missed with radiological studies. These results are very different from those of Dearlove's Australian necropsy series, but his was a retrospective study of 7,000 hospital pathology records, a method very likely to underestimate a condition as unobtrusive as diverticulosis may be. In fact, this high incidence of diverticulosis is neither unique nor recent. Watt and Marcus ${ }^{3}$ reported a British series where diverticulosis was present in 42 of 76 consecutive necropsies, while Bumm ${ }^{4}$ in Germany found an incidence of $33 \%$ in 920 necropsies over the age of 50 .

The acceptance of this high incidence of diverticular disease is necessary for a true assessment of much of the published work on colonic disease. For instance, the interesting article on colonic motility by Manousos et al. showed a marked difference in bowel motility between the normal and diverticular groups. As the normal patients were selected because of a failure to show diverticula on barium studies, it is likely that this group contained a considerable number of patients with early diverticular disease. If this is so, an even greater difference with less scatter in the normal group may exist between normal and diverticular groups. - I am, etc.,

Department of Surgery,
University of Queensland,
Brisbane, Australia.
References
Dearlove, T. P., Med. 7. Aust., 1954, 1, 470.
Hughes, L. E., and Burnett, W., Gut, 1967, 8,
199, J., and Marcus, R., 7. Path. Bact., 1964,
Watt, J9, and
88, 97.
Bumm, R., Arch. Klin. Chir., 1933, 174, 14.

\section{Dysmenorrhoea}

SIR,-I was delighted to note that in his recent letter Mr. A. Davis (11 November, p. 357) has swept away the mumbo-jumbo associated with the management of an ailment which in its acute form is quite as agonizing as biliary or renal colic-and for these we do not prescribe an aspirin and some good advice.

It is difficult to understand on what grounds lack of exercise is postulated as an aggravating factor; the worst case I ever knew was that of a jolly extravert who was always top in gymnastics and whose favourite hobby was hill climbing.

Having disposed of these antiquated ideas, Mr. Davis goes on to suggest some practical treatment; and practical help, based on some knowledge of physiology, is certainly needed for these sufferers, and more research. For many years I have found one course of premenstrual progesterone to be most effective. The patients suffered no ill effects, and many are now married with families.-I am, etc.,

Glasgow

JEAN WILSON.

\section{Point of Death}

SIR,-On 3 December I listened on Radio 4 to a "live" interview with a surgeon who had transplanted a heart. I wish to emphasize that this was not a newspaper report but a radio interview. He said that he had a recipient and that a suitable donor became available. (Usual tests satisfactory.) $\mathrm{He}$ said that the donor had multiple fractures, severe brain damage, and that the heart had stopped; and that on the heart-lung instrument the heart restarted. It was transplanted to the recipient.

During my working life as head of a department of a teaching hospital I have had considerable experience of multiple fractures and of severe brain damage. Many cases of brain damage which earlier one would have said were hopeless have been restored to a useful working life. And in heart stoppage, except for the record, it is hardly necessary to refer to the thousands of cases where the heart has stopped and been restarted, as indeed it was in the case of the donor.

Disregarding ethical questions, with which I am not competent to deal, and which will almost certainly be considered elsewhere, may I, through your columns, ask the surgeon who performed the operation one question? At what point did the donor die ?-I am, etc.,

London S.W. 3

Clive Shields.

\section{Information on Patients}

SIR,-Within the past month I have had direct requests from a medical officer of health that I should give to him information, in no way of a public health matter, regarding the health of a patient of mine who is an employee of the county council, and from a company doctor asking for information regarding the health of an employee of the company concerned. Both these requests were sent direct to me without my patients even being told that I was being approached, and both were requests for reports affecting the patients' future employment and livelihood.

That there are many members of the medical profession who have become so imbued with a bureaucratic philosophy as to regard this procedure as normal and who presumably regularly do their work in this manner without rebuff is, I am sure, a manifestation of one of the ills that beset medical practice today. One meets an increasing number of doctors who do not seem to realize or have not been taught in their medical schools that they are not cogs serving a vast impersonal bureaucratic machine, but are individual doctors practising individual medicine with individual patients to whom they owe $100 \%$ loyalty. - I am, etc.,

$$
\text { Hornsea, }
$$

